\title{
Cyclin-Dependent Kinase 5 Supports Neuronal Survival through Phosphorylation of Bcl-2
}

\author{
Zelda H. Cheung, Ke Gong, and Nancy Y. Ip \\ Department of Biochemistry, Hong Kong University of Science and Technology, Clear Water Bay, Hong Kong, China
}

\begin{abstract}
Accumulating evidence indicates that deregulation of cyclin-dependent kinase $5(\mathrm{Cdk} 5)$ activity is associated with apoptosis in various neurodegenerative disease models. Interestingly, recent studies suggest that Cdk5 may also favor neuronal survival. Nonetheless, whether $\mathrm{Cdk} 5$ is directly required for neuronal survival during development remains enigmatic. In the current study, we established the pivotal role of $\mathrm{Cdk} 5$ in neuronal survival during development by demonstrating that reduction or absence of Cdk5 activity markedly exacerbated neuronal death in cultures and in vivo. Interestingly, the antiapoptotic protein Bcl-2 (B-cell lymphoma protein 2) was identified as a novel substrate of Cdk5. We found that Cdk5-mediated phosphorylation of Bcl-2 at Ser70 was required for the neuroprotective effect of Bcl-2. Intriguingly, inhibition of this phosphorylation conferred proapoptotic property to Bcl-2. Furthermore, overexpression of a Bcl-2 mutant lacking the Cdk5 phosphorylation site abolished the protective effect of Cdk5 re-expression in $C d k 5^{-/-}$ neurons, suggesting that Ser70 phosphorylation of Bcl-2 contributed to Cdk5-mediated neuronal survival. Our observations revealed that Cdk5-mediated Bcl-2 phosphorylation is pivotal for the antiapoptotic effect of Bcl-2 and contributes to the maintenance of neuronal survival by Cdk5. Our study has also identified Cdk5 as a regulator of $\mathrm{Bcl}-2$ function in neuronal apoptosis.
\end{abstract}

Key words: apoptosis; p35; Bcl-2; Cdk5; neuronal survival; retinal ganglion cells

\section{Introduction}

Cyclin-dependent kinase 5 ( Cdk5) is a proline-directed serine/ threonine kinase that is implicated in the regulation of neuronal migration, neuronal survival, and synaptic functions. Unlike other Cdks, activation of Cdk5 requires association with two noncyclin neural-specific activators $\mathrm{p} 35$ and p39, thus restricting the activity of Cdk5 to the nervous systems (Dhavan and Tsai, 2001). Recent studies reveal that precise regulation of Cdk5 activity is critical for the maintenance of neuronal survival (Cheung and Ip, 2004). Deregulated Cdk5 activity, for example, is observed in postmortem brains of Alzheimer's disease patients and is accompanied by hyperphosphorylated Tau and accumulation of p25, a cleaved fragment of p35 (Patrick et al., 1999). Furthermore, Cdk5/p25-mediated phosphorylation of NDMA receptors and prosurvival transcription factor MEF2 (myocyte enhancer factor 2) contribute to ischemia-induced neuronal death and dopaminergic neuronal loss in models of Parkinson's disease, respectively (Gong et al., 2003; Wang et al., 2003; Tang et al., 2005; Smith et al., 2006). These observations collectively indicate that

Received Feb. 15, 2008; revised March 25, 2008; accepted April 1, 2008.

This work was supported by the Research Grants Council of Hong Kong (Hong Kong University of Science and Technology Grants 6119/04M, 6444/06M, 6431/06M, and 3/03C), the Area of Excellence Scheme of the University Grants Committee Grant AoE/B-15/01, and Hong Kong Jockey Club. N.Y.I. and Z.H.C. are a Senior Research Fellow and a Fellow of the Croucher Foundation, respectively. We thank Dr. B. Barres for the protocol on pure RGC culture, Dr. Shin-Ichi Hisanaga for recombinant Cdk5/p35, and Dr. A. B. Kulkarni and Dr. T. Curran for the Cdk5 knock-out mice. We thank Wing Hong Chin, Grace Wong, Sabrina Xu, Dr. Brenda Zhao, and Dr. Yu Pong Ng for excellent technical assistance.

Correspondence should be addressed to Prof. Nancy Y. Ip, Department of Biochemistry, Hong Kong University of Science and Technology, Clear Water Bay, Hong Kong, China. E-mail: boip@ust.hk.

DOI:10.1523/JNEUROSCI.0689-08.2008

Copyright $\odot 2008$ Society for Neuroscience $\quad$ 0270-6474/08/284872-06\$15.00/0 prolonged activation of $\mathrm{Cdk} 5$ attributable to $\mathrm{p} 25$ production $\mathrm{fa}-$ vors neuronal death in various pathological conditions and neurodegenerative diseases.

Interestingly, recent studies also suggest that Cdk5 may play a role in promoting neuronal survival. For example, ballooned neurons are evident in the spinal cord and brainstem of Cdk5deficient mice (Ohshima et al., 1996). Indeed, Cdk5 activity is important for mediating the trophic effects of neuregulin ( $\mathrm{Li}$ et al., 2003). In addition, Cdk5 counters apoptotic signals through phosphorylation and inhibition of c-Jun N-terminal protein kinase 3 (JNK3) (Li et al., 2002). Recently, reduction of Cdk5 activity is associated with neuronal death after treatment with DNA damage agent (O'Hare et al., 2005). Although these findings suggest a crucial role of Cdk5 in the maintenance of survival signals, whether Cdk5 activity is directly required for neuronal survival during development has not been thoroughly investigated. In addition, the mechanisms by which Cdk5 support neuronal survival remains essentially unknown. We thus aim to further characterize the role of Cdk5 in the life/death decision of developing neurons.

\section{Materials and Methods}

Antibodies, neuronal cultures, and transfection. Antibodies against Bcl-2 (B-cell lymphoma protein 2), Cdk5, and p35 were from Santa Cruz Biotechnology (Santa Cruz, CA). Anti-phosphoserine antibody (16B4) was from Calbiochem (La Jolla, CA). Actin and $\beta$-tubulin type III antibodies were from Sigma (St. Louis, MO). Expression vectors of p35, Cdk5, and dominant-negative $\mathrm{Cdk} 5(\mathrm{dnCdk} 5)$ were prepared as described previously (Fu et al., 2001). Flag-tagged, green fluorescent protein (GST)tagged Bcl-2 and Stealth RNA interference molecules for Cdk5 were prepared as described previously (Ng et al., 2006). Selective Cdk inhibitor, roscovitine (Ros) (Calbiochem) was used to inhibit Cdk5 activity. 
A
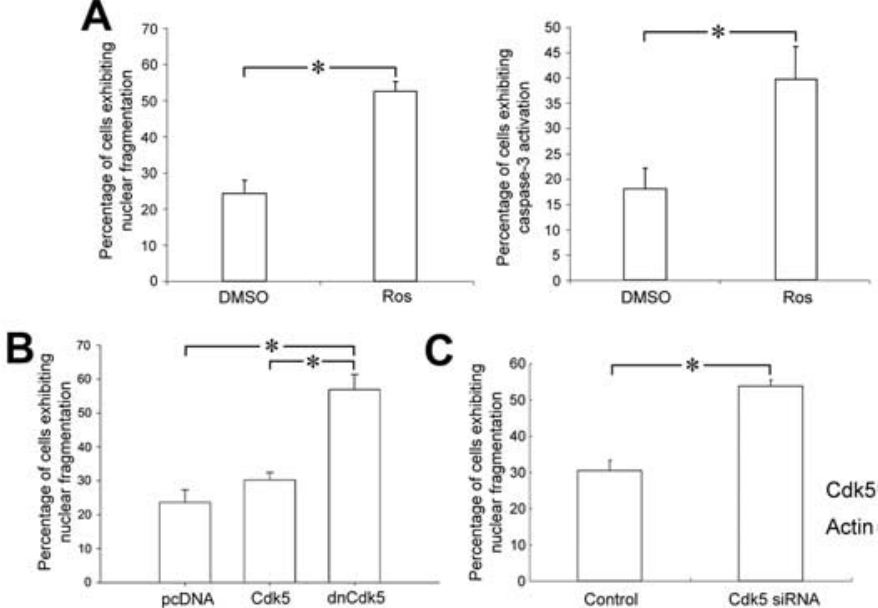

C
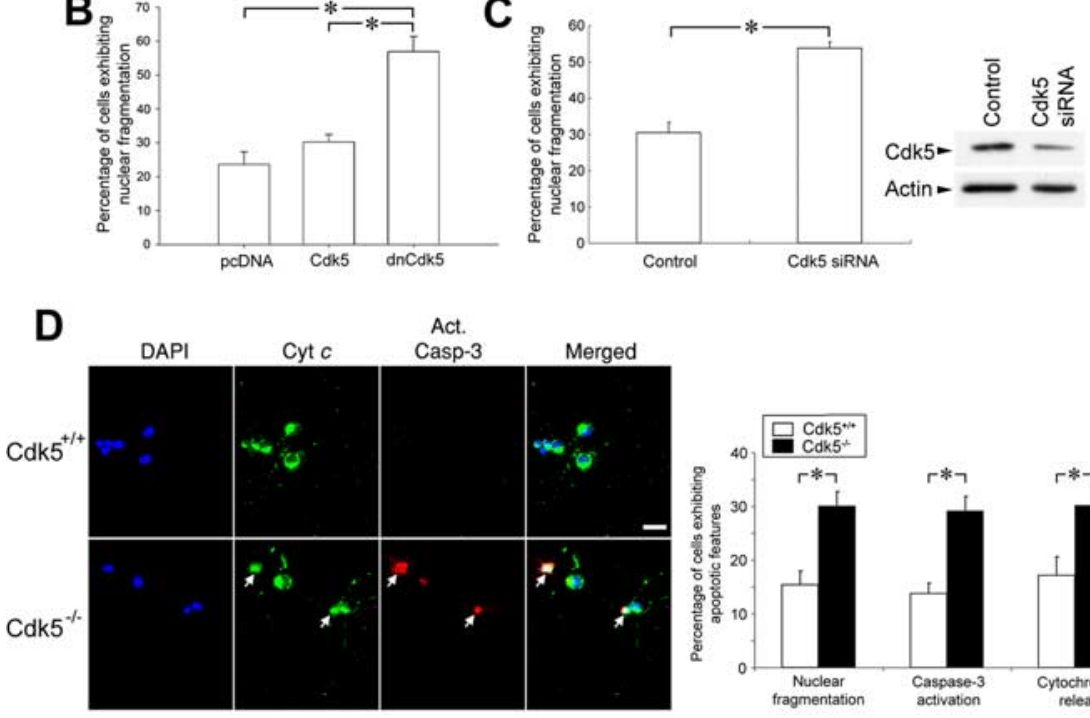

E

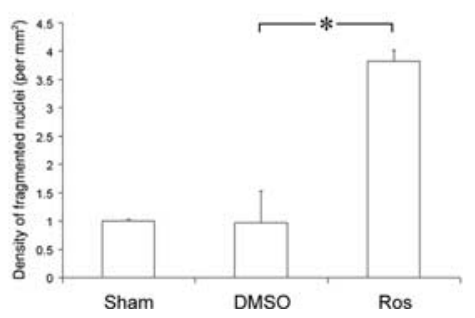

Figure 1. Cdk5 was required for neuronal survival. $\boldsymbol{A}$, Treatment with C $\mathrm{dk} 5$-selective inhibitor Ros (25 $\mu \mathrm{m})$ induced nuclear fragmentation and caspase-3 activation in pure RGC culture. $\boldsymbol{B}, 0$ verexpression of $\mathrm{dnC}$ Ck5 led to a marked increase in nuclear fragmentation in retinal neurons. $\boldsymbol{C}$, Western blot analysis demonstrated that transfection of Cdk5 siRNA, but not scrambled siRNA (control), markedly reduced C $\mathrm{dk} 5$ expression in retinal neurons. Transfection of Cdk5 siRNA increased the percentage of retinal neurons exhibiting nuclear fragmentation. $\boldsymbol{D}$, Retinal neurons taken from $\left(\mathrm{dk} 5^{-1-}\right.$ mice displayed a higher incidence of nuclear fragmentation, caspase-3 activation, and cytochrome c (Cyt c) release (arrows), as indicated by diffuse cytochrome $c$ staining. $\boldsymbol{E}$, Intravitreal injection of Ros significantly increased the incidence of nuclear fragmentation in the ganglion cell layer of adult rat retina. Scale bar, $20 \mu \mathrm{m} .{ }^{*} p<0.05$.

Cdk5 knock-out mice were kindly provided by A. B. Kulkarni (National Institutes of Health, Bethesda, MD) and T. Curran (School of Medicine, University of Pennsylvania, Philadelphia, PA). Pure retinal ganglion cell (RGC) cultures were prepared according to the protocol provided by B. Barres (Barres et al., 1988). Transfection of primary cultures was performed using calcium phosphate precipitation. Small interfering RNA (siRNA) transfection was performed with Lipofectamine 2000 transfection reagent according to the protocols of the manufacturer (Invitrogen, Carlsbad, CA). COS7 cells and HEK293T cells were maintained and transfected as described previously (Ng et al., 2006). Western blotting and immunoprecipitation was performed as described previously (Ng et al., 2006).

In vitro kinase assay, intravitreal injection, and statistical analysis. Recombinant Cdk5/p35 was kindly provided by Shin-Ichi Hisanaga (Tokyo Metropolitan University, Tokyo, Japan). In vitro kinase assay was performed as described previously (Fu et al., 2001). Intravitreal injection was performed using glass micropipettes as described previously (Cheung et al., 2004). Vehicle or Ros (estimated intravitreous concentration, $\sim 25$

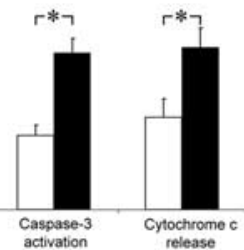

$\mu \mathrm{M})$ were injected once every $3 \mathrm{~d}$ for $6 \mathrm{~d}$. Retinas were dissected and fixed on day 7. Cell death in flat-mounted retina was quantified as described previously (Cheung et al., 2004).

Data were expressed as mean \pm SEM. Statistical significance was determined by Student's $t$ test or one-way ANOVA, followed by NewmanKeuls post hoc test with $95 \%$ confidence. $p<0.05$ was considered statistically significant.

\section{Results}

Cdk5 is required for neuronal survival

To verify whether Cdk5 directly affects neuronal survival, pure RGC cultures were prepared from postnatal day 8 (P8) rats using immunopanning. Interestingly, treatment of RGCs with Cdk5-selective inhibitor Ros for $4 \mathrm{~h}$ markedly enhanced nuclear fragmentation and caspase- 3 activation that was evident $72 \mathrm{~h}$ after the initial treatment (Fig. 1A), indicating that suppression of Cdk5 activity for $4 \mathrm{~h}$ induced delayed neuronal apoptosis in RGCs. To further substantiate the role of Cdk5 in neuronal survival, retinal neurons were transfected with wild-type or dnCdk5. Similar to the induction of apoptosis by Ros, overexpression of $\mathrm{dnCdk} 5$ led to a marked increase in nuclear fragmentation compared with those expressing wild-type Cdk5 (Fig. $1 B$ ). Furthermore, reduction of endogenous Cdk5 expression using siRNA approach also resulted in enhanced nuclear fragmentation (Fig. 1C). More importantly, retinal neurons isolated from $C d k 5^{-/-}$embryos exhibited elevated incidence of cytochrome $c$ release, caspase- 3 activation, and nuclear fragmentation (Fig. 1D). These observations strongly suggest that Cdk5 activity was required for the survival of retinal neurons.

To verify that a decrease in Cdk5 activity is sufficient to induce neuronal death in vivo, Ros was injected into the vitreous chamber of adult rats to reduce Cdk5 activity in the retina. We found that Ros injection markedly increased nuclear fragmentation in the ganglion cells layers (Fig. 1E), indicating that a reduction of Cdk5 activity was sufficient to induce apoptosis in the retina in vivo.

\section{Bcl-2 is a substrate of Cdk5}

We next proceeded to examine the potential mechanisms by which Cdk5 support neuronal survival. Because cytochrome $c$ release is markedly elevated in $C d k 5^{-1-}$ neurons, we wanted to examine whether Cdk5 affects cytochrome $c$ release through interacting with members of the $\mathrm{Bcl}-2$ family. Cytochrome $c$ release is tightly regulated by Bcl-2 family members, which includes proapoptotic members, such as Bax (Bcl-2-associated X protein) and Bak (Bcl-2-antagonist/killer), and antiapoptotic members, such as Bcl-2 and Bcl-xL (Reed, 2006). Interestingly, sequence analysis revealed that $\mathrm{Bcl}-2$ contains multiple proline-directed serine and threonine residues and one Cdk5 consensus site, S/TPXK/H/R (see Fig. $3 A$ ). This suggests that Bcl-2 may serve as a Cdk5 sub- 
strate. To examine this possibility, we first investigated whether p35, Cdk5, and Bcl-2 were localized to the same subcellular fractions. Bcl-2 is localized to the outer membrane of the mitochondria (Blagosklonny, 2001). We also found that Bcl-2 was predominantly expressed in the mitochondrial fraction of cortical neurons. Interestingly, p35 was also abundantly expressed in the mitochondrial fraction, whereas Cdk5 was detected in both the mitochondrial and cytosolic fractions (Fig. 2A). Furthermore, immunocytochemical staining revealed that p35 colocalized extensively with both Bcl-2 and mitochondrial marker subunit IV of cytochrome $c$ oxidase (COX4) in developing neurons, thus confirming the colocalization of Bcl-2 and p35 to the mitochondria (Fig. 2B). We next examined whether Bcl-2 associated with $\mathrm{p} 35$ or $\mathrm{Cdk} 5$ using lysates from HEK293T cells overexpressing p35 or Cdk5. Interestingly, whereas GST-Bcl-2 pulled down p35, and to a lesser extent Cdk5, in a GST pull-down assay (Fig. 2C), Bcl-2 only coimmunoprecipitated with p35 when both p35 and Bcl-2 were overexpressed in HEK293T cells (Fig. $2 D)$. We further verified the interaction between Bcl-2 and p35 using membrane fractions of postnatal rat brains. We found that Bcl-2 coimmunoprecipitated with p35 and Cdk5, indicating that $\mathrm{Bcl}-2$ associated with p35 and Cdk5 endogenously (Fig. 2E).

To verify whether $\mathrm{Bcl}-2$ serves as a Cdk5 substrate, GST-Bcl-2 was subjected to in vitro kinase assay using Cdk5/p35 as the kinase. Consistent with the presence of potential Cdk5 phosphorylation sites, GST-Bcl-2 was increasingly phosphorylated by $\mathrm{Cdk} 5 / \mathrm{p} 35$ with increasing concentrations of the kinase (Fig. $3 B$ ), indicating that $\mathrm{Bcl}-2$ is a substrate of $\mathrm{Cdk} 5$ / p35. We next identified the potential Cdk5 phosphorylation site(s) on Bcl-2. Phosphorylation of $\mathrm{Bcl}-2$ at the loop region has been demonstrated to modulate the neuroprotective effect of Bcl-2 (Ruvolo et al., 2001). Bcl-2 exhibits multiple proline-directed serine/threonine residues in the loop region, including Thr55, Ser70, and Ser84. In particular, a strong Cdk5 consensus site is found at Ser70 with the fourth amino acid requirement fulfilled (Fig. 3A). To identify the Cdk5 phosphorylation site(s), we generated three mutants in which Thr55 (T55A), Ser70 (S70A), or Ser84 (S84A) was mutated to alanine and examined whether Cdk5-mediated phosphorylation of $\mathrm{Bcl}-2$ was retained. Bcl-2-wild-type, Bcl-2-T55A, Bcl-2-S70A, and Bcl-2-S84A were overexpressed in COS7 cells and lysates were immunoprecipitated and subjected to in vitro kinase assay using $\mathrm{Cdk} 5 /$ p35. Interestingly, Cdk5-mediated phosphorylation of Bcl-2 was almost completely abolished when Ser70 was mutated to alanine (Fig. $3 C$ ) but not when Thr55 or Ser84 was mutated to alanine. Furthermore, when Bcl-2 and Bcl-2-S70A were overexpressed together with Cdk5 and p35 in HEK293T cells, Bcl-2, but not Bcl-2-S70A, was serine phosphorylated as detected by an antibody against phospho-
B
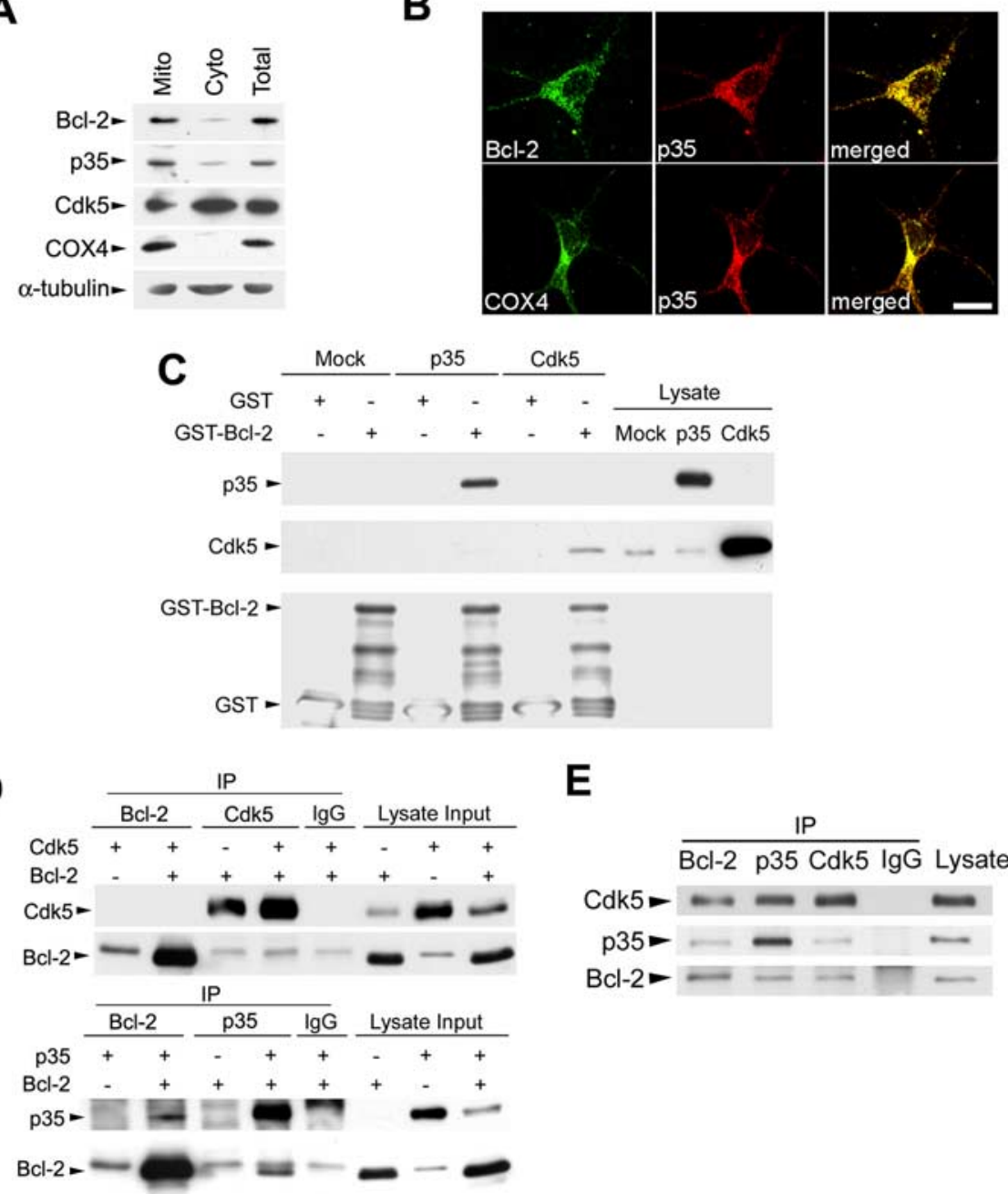

$\mathbf{E}$

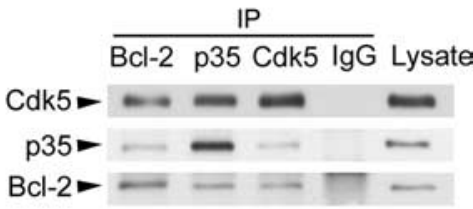

$\mathrm{Bcl}-2$

Figure 2. $B \mathrm{Bl}-2$ interacted with $\mathrm{Cdk} 5$ in adult brain. $\boldsymbol{A}, \mathrm{BCl}-2$ and $\mathrm{p} 35$ were concentrated in the mitochondrial fraction of cortical neurons, whereas Cdk5 was ubiquitously expressed. COX4 serves as a marker for the mitochondrial fraction, and

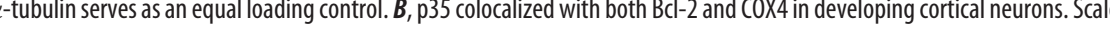
(bottom), but not Cdk5 (top), associated with Bcl-2 when overexpressed in HEK293T cells. IgG was included as a control. $\boldsymbol{E}, \mathrm{BCl}-2$ associated endogenously with $\mathrm{Cdk} 5$ and $\mathrm{p} 35$ in the membrane fraction of P7 rat brain. IP, Immunoprecipitation.

serine (Fig. 3D). These observations collectively indicate that Cdk5 phosphorylated Bcl-2 at Ser70.

To further verify whether Cdk5 phosphorylates Bcl-2 in vivo, Bcl-2 phosphorylation was examined in $C d k 5^{+/+}$and $C d k 5^{-/-}$ brains. Importantly, Bcl-2 phosphorylation was markedly reduced in $C d \mathrm{k}^{-/-}$brain, whereas Bcl-2 total levels remained rather constant, suggesting that Cdk5 phosphorylated Bcl-2 in vivo (Fig. $3 E$ ). It should be noted that a number of kinases have also been observed to phosphorylate Bcl-2, including extracellular signal-regulated kinase (Erk1/2), JNK, and p38 mitogenactivated protein kinase (MAPK) (Blagosklonny, 2001; Ruvolo et al., 2001). We thus examined the activation of these kinases in $C d k 5^{+/+}$and $C d k 5^{-/-}$brains to verify that the lack of Cdk5 did not affect Bcl-2 phosphorylation through indirect modulation of these kinases. We found that activation of Erk and p38 MAPK were both enhanced in $C d k 5^{-/-}$brain, whereas JNK activation was comparable between $C d k 5^{+/+}$and $C d k 5^{-/-}$brains (Fig. 3E). This indicates that $\mathrm{Bcl}-2$ phosphorylation was not attenuated because of inactivity of other known Bcl-2 kinases in Cdk5-deficient brain. 


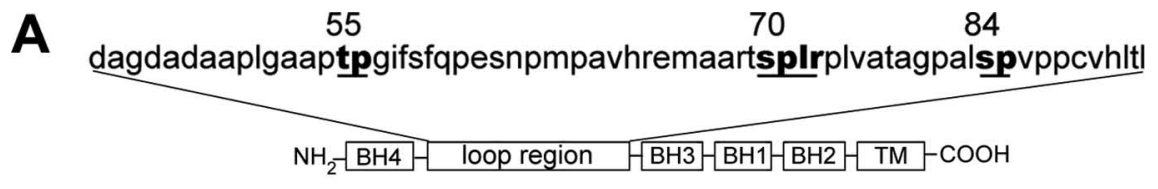

B
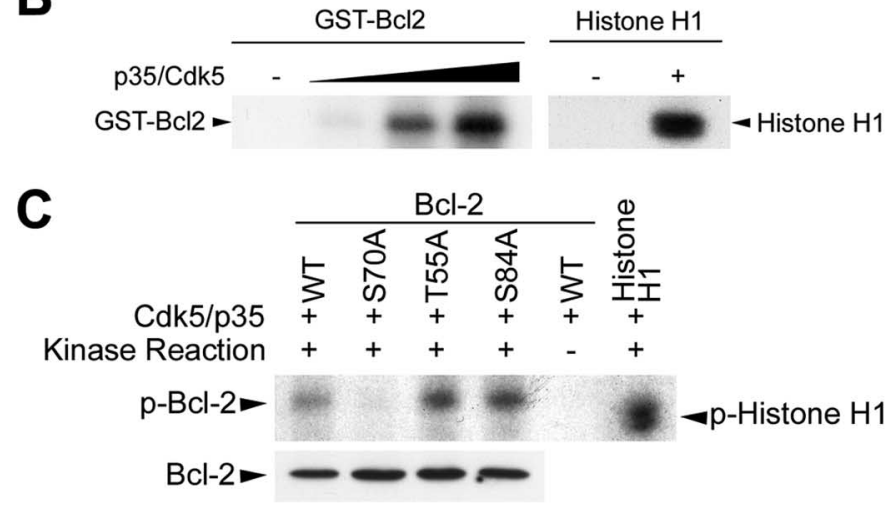

D
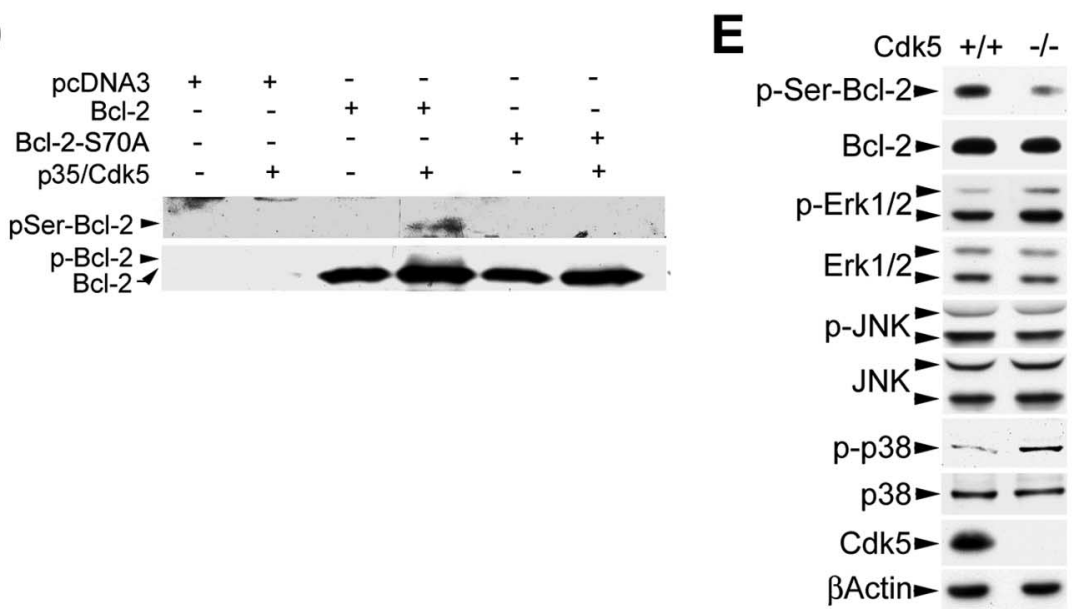

Figure 3. Cdk5 phosphorylated BCl-2 at Ser70. A, Potential Cdk5 phosphorylation sites are found at Thr55, Ser70, and Ser84 of the loop region of mouse $\mathrm{BCl}-2 . B, \mathrm{GST}-\mathrm{BCl}-2$ was phosphorylated by $\mathrm{Cdk5} / \mathrm{p} 35$ in a dose-dependent manner in in vitro kinase assay. Histone $\mathrm{H} 1$ served as a control to demonstrate activity of the Cdk5/p35 complex. C, Wild-type (WT) BCl-2, BCl-2-S70A, $\mathrm{BCl}-2-\mathrm{T55A}$, or BCl-2-S84A were subjected to in vitro kinase assay. Mutation of Ser70, but not Thr55 or Ser84, to alanine abolished phosphorylation of $\mathrm{BCl}-2$ by $\mathrm{Cdk5} / \mathrm{p} 35$. D, Bcl-2, but not $\mathrm{BCl}-2-570 \mathrm{~A}$, was serine phosphorylated (pSer-Bcl-2) in the presence of $\mathrm{Cdk} 5 / \mathrm{p} 35$. The serine-phosphorylated band corresponded to an upward shift in mobility (pBCl-2) when the blot was reprobed with $\mathrm{BCl}-2$ antibody. $E, \mathrm{BCl}-2$ phosphorylation ( $\mathrm{p}-\mathrm{BCl}-2)$ was markedly reduced in $\mathrm{Cdk}^{-/-}$brain. Activation of $\mathrm{BCl}-2$ kinases, such as Erk1/2, JNK, and p38 MAPK, was not reduced in $\mathrm{Cdk}^{-1-}$ brain.

\section{Phosphorylation of Bcl-2 at Ser70 by Cdk5 regulates if Bcl-2 exhibits antiapoptotic or proapoptotic property}

To examine whether Cdk5-mediated phosphorylation of Bcl-2 affects the antiapoptotic functions of Bcl-2, Bcl-2 and Bcl-2S70A were transfected into P8 retinal neurons to examine their effects on neuronal survival. Developmental apoptosis of retinal neurons occurs within the first 2-3 weeks after birth (Young, 1984). In agreement with this observation, $20-30 \%$ of $\mathrm{P} 8$ retinal neurons exhibited apoptotic features (Fig. 4A) (also in Fig. 1A$C)$. Overexpression of $\mathrm{Bcl}-2$ reduced the percentage of retinal neurons undergoing nuclear fragmentation by $\sim 50 \%$. Interestingly, overexpression of Bcl-2-S70A exhibited no neuroprotective effect and even slightly enhanced nuclear fragmentation in P8 retinal neurons (Fig. 4A). This suggests that phosphorylation of Bcl-2 at Ser70 is required for the antiapoptotic effect of Bcl-2.

To further characterize the involvement of $\mathrm{Cdk} 5$ in Bcl-2mediated regulation of neuronal survival, we examined the effect of Bcl-2 or Bcl-2-S70A in $\mathrm{Cdk5}^{+/+}$and $C d k 5^{-/-}$retinal neurons. Because of the exhibition of perinatal death by $C d k 5^{-1-} \mathrm{em}$ bryos, retinal neurons were isolated from embryonic day 18 embryos instead of P8 pups. Interestingly, overexpression of Bcl-2 failed to further decrease nuclear fragmentation in $\mathrm{Cdk5}^{+/+}$neurons (Fig. $4 \mathrm{~B}$ ), possibly because of a lower level of developmental apoptosis and different endogenous $\mathrm{Bcl}-2$ levels in retinal neurons at different developmental stages (Sharma, 2001). Surprisingly, we found that overexpression of Bcl-2-S70A resulted in a marked elevation of nuclear fragmentation in $\mathrm{Cdk}^{+/+}$neurons (Fig. 4B). Together with the slight elevation in neuronal apoptosis observed in Bcl-2-S70A-expressing P8 retinal neurons, these findings suggest that a $\mathrm{Bcl}-2$ mutant that is not phosphorylated at Ser70 may exhibit proapoptotic effect and suggest that Cdk5 may contribute to determining whether Bcl-2 exhibits antiapoptotic or proapoptotic property through phosphorylation at Ser 70 .

To investigate this possibility, we adopted a model in which transient overexpression of $\mathrm{Bcl}-2$ triggers apoptosis in HEK293T cells. Despite the well established role of Bcl-2 as an antiapoptotic molecule, several studies have demonstrated that transient overexpression of $\mathrm{Bcl}-2$ in cell lines such as HEK293T cells induces apoptosis (Uhlmann et al., 1998; Portier and Taglialatela, 2006). Because Cdk5 activity is absent in HEK293T cells attributable to the lack of p35 expression (Dhavan and Tsai, 2001), we hypothesized that Bcl-2 may exhibit apoptosis-inducing effect in HEK293T cells because it exists mainly as an unphosphorylated form at Ser70 in these cells. Indeed, pSer-Bcl-2 was not detected in the absence of p35/Cdk5 overexpression in HEK293T cells (Fig. $3 D$ ). To verify our hypothesis, we examined whether $\mathrm{p} 35$ overexpression, which leads to Cdk5 activation, can attenuate Bcl-2-triggered cell death in HEK293T cells. As reported previously (Uhlmann et al., 1998; Portier and Taglialatela, 2006), overexpression of Bcl-2 in HEK293T cells induced massive nuclear fragmentation (Fig. 4C). Remarkably, overexpression of p35 significantly reduced Bcl-2-induced cell death (Fig. 4C), indicating that $\mathrm{Bcl}-2$ phosphorylation by $\mathrm{Cdk} 5$ attenuated the apoptosisinducing property of Bcl-2 in HEK293T cells. Bcl-2-S70A, conversely, induced similar extent of apoptosis with or without p35 (Fig. 4C). Our findings therefore revealed that Cdk5 played an important role in modulating the precise role of Bcl-2 in apoptosis regulation. Through phosphorylation at Ser70, Cdk5 possibly regulates whether Bcl-2 exhibits proapoptotic or antiapoptotic function. Our findings also suggest that the lack of Bcl-2 phosphorylation at Ser70 in cell lines such as HEK293T cells may play a role in the induction of apoptosis by Bcl-2. 


\section{Bcl-2 phosphorylation at Ser70} contributes to the maintenance of neuronal survival by $\mathbf{C d k} 5$

We next examined whether the lack of Bcl-2 phosphorylation at Ser70 contributed to augmented neuronal death in $C d k 5^{-/-}$ mice by comparing the effect of Bcl-2 and Bcl-2-S70A overexpression in $C d k 5^{-1-}$ and $C d k 5^{+/+}$retinal neurons. Interestingly, Bcl-2 overexpression failed to exhibit any neuroprotective effect despite the extensive neuronal death observed in $C d k 5^{-/-}$neurons (Fig. $4 B$ ). Furthermore, in contrast to the marked induction of apoptosis by Bcl2-S70A in $\mathrm{Cdk}^{+/+}$neurons, Bcl-2-S70A overexpression had negligible effect on neuronal survival in $C d k 5^{-/-}$neurons (Fig. $4 B$ ). These observations suggest that $\mathrm{Bcl}-2$ loses its ability to regulate neuronal survival when Cdk5 activity is absent, thus underscoring the importance of Cdk5 in the apoptosis-regulatory role of $\mathrm{Bcl}-2$. In addition, it is interesting to note that lack of Cdk5 activity (mock-transfected Cdk5 $5^{-1-}$ neurons) and removal of the Cdk5 phosphorylation site in Bcl-2 (Bcl-2-S70Atransfected $C d k 5^{+/+}$neurons) triggered comparable levels of neuronal apoptosis, but the concurrent inhibition of Cdk 5 activity and Bcl-2 phosphorylation at Ser70 (Bcl-2-S70A-transfected Cdk5 $5^{-1-}$ neurons) had no additive effect on the induc-

tion of neuronal death (Fig. $4 \mathrm{~B}$ ). These observations are in support of the scenario in which lack of Cdk5 activity and inhibition of Bcl-2 Ser70 phosphorylation contributed to neuronal death through the same pathway. In light of the observed phosphorylation of Bcl- 2 at Ser70 by Cdk 5 and the identification of Cdk 5 as an important kinase of Bcl-2 in vivo (Fig. $3 E$ ), we believe that the lack of Bcl-2 phosphorylation at Ser70 in C $d k 5^{-/-}$neurons contributed, at least in part, to the compromised neuronal survival in $C d k 5^{-1-}$ neurons.

To further consolidate the involvement of Ser70 phosphorylation of $\mathrm{Bcl}-2$ in the maintenance of neuronal survival by Cdk5, we attempted to reverse neuronal death in $C d k 5^{-/-}$neurons through the overexpression of Cdk5. As expected, overexpression of Cdk5 markedly reduced nuclear fragmentation in $C d k 5^{-/-}$ neurons (Fig. 4D). Interestingly, although cotransfection of Bcl-2 did not offer additional neuroprotection, concomitant overexpression of Bcl-2-S70A abolished the neuroprotective effect of Cdk5 and even further enhanced neuronal apoptosis compared with mock-transfected $C d k 5^{-/-}$neurons (Fig. $4 D$ ). Our observation therefore revealed that Ser70 phosphorylation of Bcl-2 was crucial for Cdk5 overexpression-mediated neuronal survival in $C d k 5^{-/-}$neurons. In addition, similar to what was observed in Figure $4, A$ and $B$, mutation of the Cdk5 phosphorylation site conferred proapoptotic ability to Bcl-2 (Fig. $4 D$ ), further supporting the notion that phosphorylation of Bcl-2 by Cdk5 modulates the apoptosis regulatory role of Bcl-2. Collectively, our findings suggest that the lack of Bcl-2 Ser70 phosphorylation likely contributes to the enhanced developmental apoptosis exhibited by $C d k 5^{-/-}$neurons and reveal an unexpected role of $\mathrm{Cdk} 5$ as a regulator of the antiapoptotic property of $\mathrm{Bcl}-2$, where
B
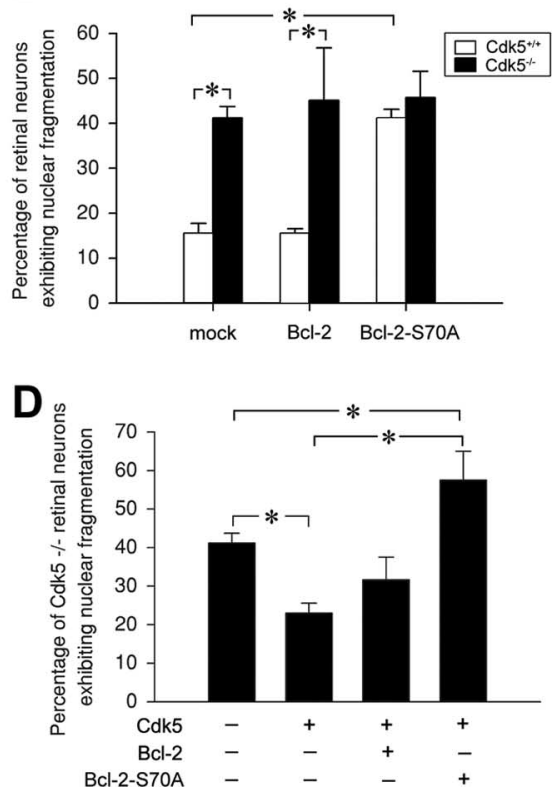

Figure 4. Phosphorylation of $\mathrm{BCl}-2$ at Ser70 determined whether $\mathrm{BCl}-2$ exhibited proapoptotic or antiapoptotic function and

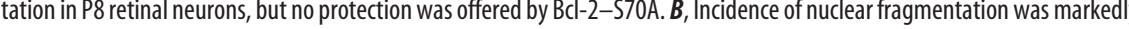

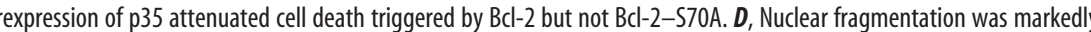
reduced by $\mathrm{Cdk} 5$ overexpression in $\mathrm{C} \mathrm{k} 5^{-/-}$neurons. Coexpression of $\mathrm{BCl}-2-\mathrm{S} 70 \mathrm{~A}$ abolished the neuroprotective effect of $\mathrm{Cdk} 5$

lack of Cdk5 phosphorylation appears to turn Bcl-2 into a proapoptotic protein.

\section{Discussion}

Numerous reports have documented phosphorylation of Bcl-2 by a number of kinases, such as receptor tyrosine kinase and MAPK kinases (Blagosklonny, 2001; Ruvolo et al., 2001). Here we have identified Cdk5 as a novel Bcl-2 kinase that phosphorylates $\mathrm{Bcl}-2$ at Ser70. Importantly, marked reduction of Bcl-2 phosphorylation was observed in $C d k 5^{-/-}$brain, thus underscoring the importance of $\mathrm{Cdk} 5$ in $\mathrm{Bcl}-2$ phosphorylation in vivo. Indeed, activation of other kinases that have been demonstrated to phosphorylate Bcl-2 at Ser70, such as Erk1/2, JNK, and p38 MAPK, were elevated or relatively unchanged in $C d k 5^{-/-}$brains compared with $C d k 5^{+/+}$brains. Nonetheless, the in vivo involvement of these kinases in Bcl-2 phosphorylation in the developing brain remains primarily enigmatic. Our observations suggest that Cdk5 serves as one of the important kinases for Bcl-2 phosphorylation in the developing nervous system in vivo. Furthermore, inhibiting Cdk5-mediated Bcl-2 phosphorylation abolished the maintenance of neuronal survival by exogenous expression of Cdk5 in $C d k 5^{-/-}$neurons, suggesting that the reduced Bcl-2 phosphorylation likely contributes to the compromised survival of $C d k 5^{-\prime-}$ neurons.

Despite the myriad of kinases that have been demonstrated to phosphorylate Bcl-2, whether phosphorylation of Bcl-2 enhances or diminishes the antiapoptotic roles of $\mathrm{Bcl}-2$ remains controversial. Recent literature suggests that, although phosphorylation of $\mathrm{Bcl}-2$ at Ser70 alone is required for the antiapoptotic effect of Bcl-2, phosphorylation of Bcl-2 at multiple sites (Ser70, Ser87, and Thr69 of human Bcl-2) turns Bcl-2 into a proapoptotic mol- 
ecule (Ruvolo et al., 2001). In the current study, we found that Bcl-2 was phosphorylated by Cdk 5 only at Ser70. Consistent with previous observations, inhibition of $\mathrm{Bcl}-2$ phosphorylation at Ser70 essentially abolished the antiapoptotic effect of Bcl-2. Unexpectedly, we also revealed that a mutant lacking the C $\mathrm{dk} 5$ phosphorylation site not only exhibited no neuroprotective effect, but instead exacerbated neuronal death. Our observations therefore suggest that Cdk5 may take part in determining whether Bcl-2 exhibits antiapoptotic or proapoptotic role through Ser70 phosphorylation of Bcl-2. Indeed, previous studies have demonstrated that Bcl-2 can exhibit proapoptotic function under certain conditions. Aside from phosphorylation of Bcl-2 at multiple sites (Ruvolo et al., 2001), localization of Bcl-2 to the nucleus also triggers apoptosis (Portier and Taglialatela, 2006). Furthermore, transient overexpression of $\mathrm{Bcl}-2$ and caspase-mediated cleavage of Bcl-2 have both been observed to induce cell death (Cheng et al., 1997; Uhlmann et al., 1998). Association of Bcl-2 with orphan nuclear receptor Nur77 also turns Bcl-2 into a proapoptotic protein (Lin et al., 2004). Whether Cdk5-mediated Bcl-2 phosphorylation acts together with these known pathways to trigger conversion of Bcl-2 into a killer or via a currently unknown mechanism remains to be determined. It is plausible that Cdk5mediated phosphorylation of Bcl-2 at Ser70 may affect its subcellular localization or its cleavage by caspases. Alternatively, this phosphorylation may directly affect its association with other Bcl-2 family members, thereby favoring or hindering apoptosis. Additional studies will be required to delineate these possibilities.

Together, our data reveal that $\mathrm{Bcl}-2$ phosphorylation by Cdk 5 is important for the antiapoptotic property of Bcl-2 and that this phosphorylation contributes to Cdk5-mediated maintenance of neuronal survival. Identification of Bcl- 2 as a Cdk 5 substrate provides an additional mechanism by which Cdk5 affects neuronal survival. Furthermore, our findings support the notion that precise regulation of Cdk5 activity is critical for neuronal survival. In light of the pivotal role of Cdk5 in neurodegenerative diseases and the essential involvement of $\mathrm{Bcl}-2$ in regulating neuronal survival, it will be interesting to further delineate the significance of this crosstalk during traumatic injury and neurodegenerative diseases in vivo.

\section{References}

Barres BA, Silverstein BE, Corey DP, Chun LL (1988) Immunological, morphological, and electrophysiological variation among retinal ganglion cells purified by panning. Neuron 1:791-803.

Blagosklonny MV (2001) Unwinding the loop of Bcl-2 phosphorylation. Leukemia 15:869-874.

Cheng EH, Kirsch DG, Clem RJ, Ravi R, Kastan MB, Bedi A, Ueno K, Hardwick JM (1997) Conversion of Bcl-2 to a Bax-like death effector by caspases. Science 278:1966-1968.

Cheung ZH, Ip NY (2004) Cdk5: mediator of neuronal death and survival. Neurosci Lett 361:47-51.

Cheung ZH, Chan YM, Siu FK, Yip HK, Wu W, Leung MC, So KF (2004)
Regulation of caspase activation in axotomized retinal ganglion cells. Mol Cell Neurosci 25:383-393.

Dhavan R, Tsai LH (2001) A decade of CDK5. Nat Rev Mol Cell Biol 2:749-759.

Fu AK, Fu WY, Cheung J, Tsim KW, Ip FC, Wang JH, Ip NY (2001) Cdk5 is involved in neuregulin-induced AChR expression at the neuromuscular junction. Nat Neurosci 4:374-381.

Gong X, Tang X, Wiedmann M, Wang X, Peng J, Zheng D, Blair LA, Marshall J, Mao Z (2003) Cdk5-mediated inhibition of the protective effects of transcription factor MEF2 in neurotoxicity-induced apoptosis. Neuron 38:33-46.

Li BS, Zhang L, Takahashi S, Ma W, Jaffe H, Kulkarni AB, Pant HC (2002) Cyclin-dependent kinase 5 prevents neuronal apoptosis by negative regulation of c-Jun N-terminal kinase 3. EMBO J 21:324-333.

Li BS, Ma W, Jaffe H, Zheng Y, Takahashi S, Zhang L, Kulkarni AB, Pant HC (2003) Cyclin-dependent kinase-5 is involved in neuregulin-dependent activation of phosphatidylinositol 3-kinase and Akt activity mediating neuronal survival. J Biol Chem 278:35702-35709.

Lin B, Kolluri SK, Lin F, Liu W, Han YH, Cao X, Dawson MI, Reed JC, Zhang XK (2004) Conversion of Bcl-2 from protector to killer by interaction with nuclear orphan receptor Nur77/TR3. Cell 116:527-540.

Ng YP, Cheung ZH, Ip NY (2006) STAT3 as a downstream mediator of Trk signaling and functions. J Biol Chem 281:15636-15644.

O’Hare MJ, Kushwaha N, Zhang Y, Aleyasin H, Callaghan SM, Slack RS, Albert PR, Vincent I, Park DS (2005) Differential roles of nuclear and cytoplasmic cyclin-dependent kinase 5 in apoptotic and excitotoxic neuronal death. J Neurosci 25:8954-8966.

Ohshima T, Ward JM, Huh CG, Longenecker G, Veeranna, Pant HC, Brady RO, Martin LJ, Kulkarni AB (1996) Targeted disruption of the cyclindependent kinase 5 gene results in abnormal corticogenesis, neuronal pathology and perinatal death. Proc Natl Acad Sci USA 93:11173-11178.

Patrick GN, Zukerberg L, Nikolic M, de la Monte S, Dikkes P, Tsai LH (1999) Conversion of p 35 to p25 deregulates Cdk5 activity and promotes neurodegeneration. Nature 402:615-622.

Portier BP, Taglialatela G (2006) Bcl-2 localized at the nuclear compartment induces apoptosis after transient overexpression. J Biol Chem 281:40493-40502.

Reed JC (2006) Proapoptotic multidomain Bcl-2/Bax-family proteins: mechanisms, physiological roles, and therapeutic opportunities. Cell Death Differ 13:1378-1386.

Ruvolo PP, Deng X, May WS (2001) Phosphorylation of Bcl2 and regulation of apoptosis. Leukemia 15:515-522.

Sharma RK (2001) Expression of Bcl-2 during the development of rabbit retina. Curr Eye Res 22:208-214.

Smith PD, Mount MP, Shree R, Callaghan S, Slack RS, Anisman H, Vincent I, Wang X, Mao Z, Park DS (2006) Calpain-regulated p35/cdk5 plays a central role in dopaminergic neuron death through modulation of the transcription factor myocyte enhancer factor 2. J Neurosci 26:440-447.

Tang X, Wang X, Gong X, Tong M, Park D, Xia Z, Mao Z (2005) Cyclindependent kinase 5 mediates neurotoxin-induced degradation of the transcription factor myocyte enhancer factor 2. J Neurosci 25:4823-4834.

Uhlmann EJ, Subramanian T, Vater CA, Lutz R, Chinnadurai G (1998) A potent cell death activity associated with transient high level expression of BCL-2. J Biol Chem 273:17926-17932.

Wang J, Liu S, Fu Y, Wang JH, Lu Y (2003) Cdk5 activation induces hippocampal CA1 cell death by directly phosphorylating NMDA receptors. Nat Neurosci 6:1039-1047.

Young RW (1984) Cell death during differentiation of the retina in the mouse. J Comp Neurol 229:362-373. 